\title{
Medidas provisionales adoptadas por la Corte Interamericana de Derechos Humanos en el asunto B. con El Salvador y el fortalecimiento de la protección de los derechos reproductivos en el sistema interamericano
}

Inter American Court of Human Rights provisional measures adopted in B. v. El Salvador case and the strengthening of reproduction rights in the inter American system

\author{
Mónica Arango Olaya \\ Centro de Derechos Reproductivos, Colombia
}

Abogada de la Universidad de los Andes (Bogotá, Colombia), con una maestría en Derecho en la Universidad de Harvard. Es Directora Regional para América Latina y el Caribe del Centro de Derechos Reproductivos. Antes de incorporarse al Centro de Derechos Reproductivos, trabajó en la Corte Constitucional de Colombia, en el Congreso de Colombia y en el CIJUS de la Universidad de los Andes. Ha publicado artículos sobre derecho constitucional, derecho internacional y derechos reproductivos.

marango@reprorights.org

\section{RESUMEN}

Este artículo aborda las medidas provisionales dictadas por la Corte Interamericana de Derechos Humanos respecto de El Salvador y a favor de "B" en mayo de 2013. Se hace un repaso por las características y la naturaleza de las medidas provisionales que puede dictar la Corte Interamericana, con el objeto de entender el valor del pronunciamiento en este caso. Además, se analizan las principales implicancias de esta resolución para la comprensión del contenido y alcance del derecho a la vida, a la salud e integridad personal, así como para la consolidación del reconocimiento de los derechos reproductivos en el Sistema Interamericano.

Palabras clave: Medidas provisionales - derechos reproductivos- derecho a la vida- derecho a la salud.

\begin{abstract}
This article talks about provisional measures issued by the Inter American Court of Human Rights in the B. v. El Salvador case in May, 2013. It describes the characteristics and nature of the Courts provisional measures in order to understand their value in this case. Furthermore, the article analyses the main implications of this case towards the understanding of the content and scope of the right to life, to health and personal integrity, as well as for the consolidation of the recognition of reproductive rights in the Inter American System.
\end{abstract}

Key words: Provisional measures - reproductive rights - right to life- right to health. 


\section{Introducción}

Las medidas provisionales otorgadas por la Corte Interamericana de Derechos Humanos (Corte IDH) en mayo del $2013^{1}$ a favor de Beatriz (B.) en El Salvador, tienen importantes consecuencias para la consolidación del reconocimiento de los derechos reproductivos en el Sistema Interamericano (SIDH). Este asunto trata sobre Beatriz, una mujer joven con una enfermedad grave, que se encontraba embarazada de un feto diagnosticado incompatible con la vida extrauterina. Debido a su enfermedad, el embarazo le ocasionaba una amenaza a su vida y a su salud. Si bien el diagnóstico médico le indicaba la terminación del embarazo, la normativa salvadoreña que penaliza el aborto en todas las circunstancias no se lo permitía. Las medidas provisionales otorgadas por la Corte IDH tutelaron sus derechos a la vida, a la integridad personal y a la salud, ordenando que se realizaran todos los tratamientos médicos indicados para proteger sus derechos.

Con la dictación de estas medidas provisionales, la Corte IDH por primera vez se pronuncia sobre la interrupción del embarazo en un contexto de criminalización total del aborto. En este sentido, la decisión contiene al menos tres aspectos que merecen atención. Primero, las medidas reiteran el razonamiento que la Corte IDH había desarrollado en el caso Artavia Murillo vs. Costa Rica ${ }^{2}$ sobre el alcance del artículo 4.1 de la Convención Americana sobre Derechos Humanos $(\mathrm{CADH})$. Segundo, las medidas reconocen explícitamente la dimensión psíquica del derecho a la salud, lo que también reitera el alcance de su protección desde el derecho a la integridad personal y; por último, las medidas solidifican el reconocimiento de los derechos reproductivos en el SIDH.

Este artículo abordará el valor de las medidas cautelares y provisionales para comprender la trascendencia de su contenido para la jurisprudencia en el SIDH. Asimismo, se dará cuenta de manera general de los hechos que dieron origen a la dictación de las medidas cautelares por la Corte IDH, para finalmente abordar las principales implicancias de esta resolución de acuerdo a los tres puntos planteados.

\section{Las medidas provisionales y cautelares en el SIDH}

En el SIDH existen dos tipos de medidas de carácter preventivo que buscan proteger los derechos humanos ante la inminencia de un daño: las medidas cautelares, emitidas por la Comisión Interamericana de Derechos Humanos $(\mathrm{CIDH})$ y las medidas provisionales, que son dictadas por la Corte IDH. Estas medidas tienen dos características esenciales reconocidas por la jurisprudencia. Por una parte, son cautelares, lo que implica que tienen como objetivo preservar una situación jurídica, y por otra, son tutelares, por cuanto protegen derechos humanos ${ }^{3}$. Es posible adoptar cualquiera de las medidas sin necesidad de que la Corte IDH o la CIDH estén conociendo del asunto particular y en cualquier momento, es decir, no están sujetas a una determinada oportunidad procesal. Si bien las dos medidas son similares respecto a su objetivo, contienen algunas diferencias sustanciales.

Las medidas cautelares tienen origen en el reglamento de la $\mathrm{CIDH}^{4}$ y están reguladas por el

Corte IDH. Asunto B., medidas provisionales respecto de El Salvador, 29 de mayo de 2013. Disponible [en línea] <http://www.corteidh.or.cr/docs/medidas/B_se_01.pdf> [consulta: 16 de enero de 2014].

Corte IDH. Caso Artavia Murillo y otros (Fertilización in vitro) vs. Costa Rica. Excepciones Preliminares, Fondo, Reparaciones y Costas. Sentencia de 28 noviembre de 2012. Serie C No. 264.

Corte IDH. Caso del Periódico "La Nación", medidas provisionales respecto de Costa Rica, 6 de diciembre de 2001, considerando cuarto. Véase también: PALMA, Francisco y NOGUEIRA, Andrés. Medidas provisionales en la Corte Interamericana de Derechos Humanos. Santiago: Editorial Librotecnia, 2013.

Reglamento de la $\mathrm{CIDH}$, aprobado por la Comisión en su $137^{\circ}$ período ordinario de sesiones, celebrado del 28 
artículo 25. La CIDH, a través de las medidas, por iniciativa propia o a solicitud de parte, le indica a un Estado que debe adoptar una serie de acciones para proteger los derechos humanos protegidos por la CADH y así evitar un daño inminente. Los requisitos para su adopción son los mismos que para las medidas provisionales: i) urgencia, ii) gravedad y iii) necesidad para evitar daños irreparables. No obstante, no requieren del requisito de "extrema" gravedad, necesario para las medidas provisionales ${ }^{5}$.

Las medidas provisionales tienen origen convencional en el artículo 63.2 de la CADH, que establece que en "casos de extrema gravedad y urgencia, y cuando se haga necesario evitar daños irreparables a las personas, la Corte, en los asuntos que esté conociendo, podrá tomar las medidas provisionales que considere pertinentes. Si se tratare de asuntos que aún no estén sometidos a su conocimiento, podrá actuar a solicitud de la Comisión".

El artículo 27 del Reglamento de la Corte IDH reitera la necesidad de que existan los tres elementos mencionados para otorgar las medidas, a saber: i) extrema gravedad, ii) urgencia, y iii) cuando sea necesario para evitar daños irreparables a las personas. Para decretar las medidas, la Corte IDH debe verificar la efectividad de las acciones estatales frente a la situación, y el grado de desprotección en que quedarían las personas sobre las que se solicita las medidas, de no ser adoptadas.

Las medidas provisionales exigen a los Estados parte adoptar acciones (positivas o negativas) y son vinculantes para los Estados, de la misma forma que lo son las decisiones de fondo y las opiniones consultivas que dicta la Corte IDH. La vinculatoriedad de las medidas se sostiene principalmente en dos fuentes. La primera, de carácter convencional, toda vez que su existencia se deriva explícitamente de la CADH (artículo 63.2), así como de la obligación que se desprende del artículo 1.1 de la $\mathrm{CADH}^{6}$ (respeto, garantía y no discriminación); y la segunda, de la clasificación de las medidas como actos jurisdiccionales, ya que de acuerdo con el artículo 68.1 de la $\mathrm{CADH}$, todos los actos jurisdiccionales dictados por la Corte IDH -sin distinción- tienen carácter obligatorio $^{7}$. En este sentido, que el valor jurídico de las medidas provisionales sea equiparado a las decisiones de fondo de la Corte IDH, determina que las medidas tengan la misma capacidad de contribuir al corpus iuris de los precedentes de la Corte IDH y de definir el alcance de los derechos protegidos por la CADH. Así, el precedente dictado por la Corte IDH en el asunto de Beatriz, contribuye efectivamente al desarrollo del contenido del derecho a la vida, a la integridad personal (salud) y al reconocimiento de los derechos reproductivos en el SIDH.

de octubre al 13 de noviembre de 2009 y modificado el 2 de septiembre de 2011 en su $147^{\circ}$ período ordinario de sesiones, celebrado del 8 al 22 de marzo de 2013, para su entrada en vigor el $1^{\circ}$ de agosto de 2013.

5 REY, Ernesto y REY, Ángela. "Medidas cautelares y medidas provisionales ante la Comisión y la Corte Interamericana de Derechos Humanos". Revista Jurídica Universidad de Ciencias Empresariales y Sociales, No.14, 2010, pp.127-193.

6 REY, Ernesto y REY, Ángela. Medidas provisionales y medidas cautelares en el sistema interamericano de derechos humanos. México: Instituto Interamericano de Derechos Humanos, 1996 y GONZALEZ, Felipe. "Las medidas urgentes en el Sistema Interamericano de Derechos Humanos". Revista Sur, No.13, 2010.

"El artículo 68.1 de la Convención hace referencia a la obligatoriedad de las decisiones de la Corte sin distinguir su carácter de fallos, medidas provisionales u otro tipo de resoluciones, por lo cual es extensible a cualquier tipo de resolución que emita la Corte que conlleve en forma explícita algún tipo de obligación para el Estado. En el caso Liliana Ortega y otras contra Venezuela, ante la renuencia del gobierno de dar efectivo cumplimiento a las diversas medidas provisionales ordenadas, en forma contundente resolvió: '1. Declarar que el Estado de Venezuela, por haber reconocido su competencia, está obligado a cumplir las decisiones de la Corte [...], la que tiene el poder, inherente a sus atribuciones, de supervisar el cumplimiento de las mismas. 2. Declarar igualmente que el Estado de Venezuela tiene la obligación de implementar las medidas provisionales ordenadas por la Corte". En: REY, Ernesto y REY, Ángela. Medidas provisionales y medidas cautelares... op.cit, p. 200. 


\section{El caso de Beatriz}

El 29 de mayo de 2013, la Corte IDH decretó medidas provisionales a favor de Beatriz en El Salvador, una mujer de 22 años diagnosticada con lupus eritematoso discoide agravado con nefritis lúpica. Con trece semanas de embarazo, los médicos le anunciaron que el feto que gestaba era anencefálico, una anomalía incompatible con la vida extrauterina. El procedimiento médico recomendado para evitar una muerte materna era la finalización de la gestación. Sin embargo, dada la penalización absoluta del aborto en El Salvador, no era posible realizar el procedimiento.

El 11 de abril de 2013 se presentó un recurso de amparo ante la Sala de lo Constitucional de la Corte Suprema de Justicia, en contra del Director, el Jefe de la Unidad Jurídica y el Jefe de Servicio de Perinatología del Hospital Nacional, con el fin de que se protegiera el derecho a la vida y a la salud de Beatriz. El 17 de abril del 2013, la Sala de lo Constitucional admitió el recurso de amparo y resolvió la adopción de medidas cautelares "a fin de que las autoridades demandadas garanticen el derecho a la vida y a la salud, tanto física como mental, de la señora B., brindando el tratamiento médico necesario e idóneo para la preservación de tales derechos, mientras se tramita el amparo" ${ }^{\prime \prime}$. De acuerdo a esta decisión, Beatriz fue internada en el Hospital Nacional para recibir cuidados médicos, pero no se le realizó la terminación del embarazo.

El 18 y 14 de abril de 2013 la CIDH recibió información sobre los hechos y el 29 de abril solicitó al Estado de El Salvador "la adopción de medidas cautelares para proteger la vida, la integridad personal y la salud de B". La CIDH fundamentó su decisión en las recomendaciones del Comité Médico del Hospital Nacional, el diagnóstico del feto, la falta de una decisión de la Sala Constitucional y las consecuencias del paso del tiempo en la situación de Beatriz.

Posteriormente, el Estado remitió a la CIDH información en la que señalaba que Beatriz se encontraba estable y no tenía riesgo inminente de muerte, por lo que no se le había realizado el tratamiento indicado. Las representantes elevaron una solicitud a la CIDH para que solicitara medidas provisionales, pues el peligro inminente persistía.

En mayo del 2013, la CIDH sometió ante la Corte IDH el caso de Beatriz, en el marco de una solicitud de medidas provisionales. El 28 de mayo, la Sala Constitucional declaró "sin lugar la petición formulada" y "no ha lugar el amparo promovido" por la señora B. Para la Sala Constitucional, el asunto sometido a su consideración se trataba de un problema de colisión entre los derechos a la vida y a la salud de Beatriz, y el derecho a la vida del no nacido. La Sala consideró que el derecho a la vida humana no era un derecho absoluto. No obstante, indicó que debía realizarse un ejercicio de proporcionalidad, en el que se brindara "al binomio madre-hijo equivalentes oportunidades de goce del referido derecho fundamental"9.

La Corte IDH verificó los hechos sometidos a su conocimiento y la concurrencia de los tres elementos esenciales para decretar medidas provisionales: extrema gravedad, urgencia y la posibilidad de un daño irreparable, determinando decretar medidas provisionales para salvaguardar los derechos a la vida, integridad personal y a la salud de Beatriz.

Para la Corte IDH, la gravedad del estado de salud fue comprobada por todos los estudios médicos presentados, los que indicaban que la mayor causa de mortalidad materna en pacientes embarazadas concordaban con el diagnostico de LES y nefritis lúpica.

Corte IDH. Asunto B., op.cit, párr.2 (V).

Ibídem, párr. 10 (V). 
Sobre la urgencia, la Corte IDH constató que si bien en determinados momentos Beatriz se encontraba estable, el dictamen del médico tratante establecía que "a pesar de que la paciente se encuentra estable de su enfermedad [...] debido a los cambios fisiológicos propios del embarazo aunado a la historia natural de la enfermedad de base, podría presentarse crisis en cualquier momento, volviéndose impredecible en qué instante presentará una emergencia médica"10.

En cuanto al daño irreparable, la Corte IDH verificó los riesgos que el embarazo de un feto anecefálico podían conllevar en su salud, tales como "hemorragia obstétrica grave, agravamiento del lupus, empeoramiento de su falla renal, pre eclampsia grave" ${ }^{\prime 11}$. Igualmente, consideró el estado emocional de Beatriz al determinar que "además del daño físico que podría producirse en la señora B., el Tribunal resalta que también se estaría poniendo en peligro su salud mental $[\ldots]^{\prime \prime 12}$. De esta forma, la Corte IDH determinó que se verificaba el riesgo del daño irreparable en la vida e integridad tanto física como mental de Beatriz.

En base a estas consideraciones, la Corte IDH requirió al Estado de El Salvador a que:

[...] adopte y garantice, de manera urgente, todas las medidas que sean necesarias y efectivas para que el grupo médico tratante de la señora B. pueda adoptar, sin interferencia alguna, las medidas médicas que se consideren oportunas y convenientes para asegurar la debida protección de los derechos consagrados en los artículo 4 y 5 de la Convención Americana y, de este modo, evitar daños que pudiesen llegar a ser irreparables a los derechos a la vida y a la integridad personal y a la salud de las señora B., conforme a los párrafos considerativos 11 a 17 de la presente Resolución ${ }^{13}$.

El 3 de junio de 2013 se llevó a cabo lo dispuesto en la resolución de medidas provisionales (a través de la realización de una cesárea), después de la cual el feto no sobrevivió. El 19 de agosto de 2013 la Corte IDH levantó las medidas provisionales.

\section{Las consecuencias de las medidas provisionales dictadas por la Corte IDH}

\subsection{Contenido y alcance del artículo 4.1 de la CADH}

La tutela efectiva del derecho a la vida de Beatriz y el razonamiento de la Corte IDH para Ilegar a esa conclusión, reitera el alcance que la Corte IDH le dio al artículo 4.1 de la CADH en el caso Artavia Murillo vs. Costa Rica. En esa oportunidad, la Corte estableció que los embriones no podían ser entendidos bajo la protección del artículo 4.1 de la CADH, pero además indicó que "es posible concluir de las palabras 'en general' que la protección del derecho a la vida con arreglo a dicha disposición no es absoluta, sino es gradual e incremental según su desarrollo, debido a que no constituye un deber absoluto e incondicional, sino que implica entender la procedencia de excepciones a la regla general"14.

En efecto, una lectura de las medidas provisionales podría ser que la Corte IDH en el asunto de Beatriz, al requerir al Estado para que el personal médico adoptara todas las medidas necesarias que considerara pertinentes para tutelar el derecho a la vida de la mujer, y no mencionar dentro de las mismas ninguna medida que tutele la protección del derecho a la vida del no nacido, hizo

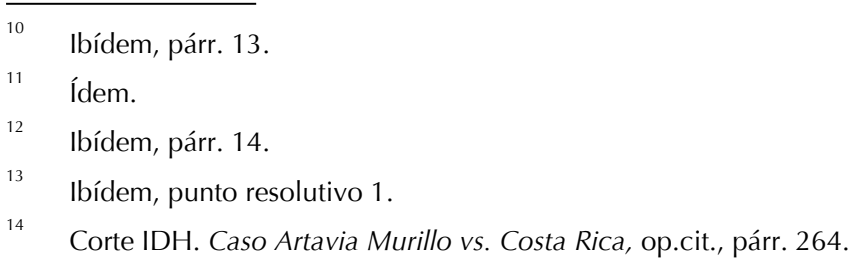


implícitamente un balance de la protección de derechos e intereses jurídicos en juego, en el que prevalece la protección a la vida de la mujer. La determinación de esta protección reitera el razonamiento de la Corte IDH en el caso Artavia Murillo, ya que aborda la protección del derecho a la vida respecto del no nacido de manera incremental y gradual, teniendo en consideración los otros derechos de la mujer en juego. Esta tesis implica que pueden existir situaciones donde la Corte IDH, de acuerdo al desarrollo gradual e incremental del no nacido, admitiera un balance de intereses jurídicos con un resultado diferente.

Sin embargo, otra lectura posible de este razonamiento, es que la Corte IDH solo encontró en los derechos de Beatriz bienes jurídicos tutelables relevantes para su consideración, en el marco particular de las medidas provisionales (que implican la imposibilidad de tomar determinaciones sobre el fondo del asunto). Lo anterior podría significar que la falta de bienes jurídicos tutelables del no nacido implica que no existe un derecho a la vida que tutelar, lo que haría innecesaria la ponderación.

Las medidas requeridas por la Corte IDH comprenden una protección "coadyudante y complementaria" a las medidas internas para buscar la mayor protección de la víctima. En este caso, las medidas internas sobrepusieron la protección del que está por nacer sobre la vida de Beatriz al denegar el recurso de amparo, por considerar que su vida no estaba en riesgo. Esa decisión otorga un valor absoluto a la vida del que está por nacer y anula no solo el derecho a la vida y a la salud de la mujer embarazada, sino también sus derechos a la integridad personal, a la autodeterminación, y a la privacidad, entre otros. Por otra parte, las medidas otorgadas por la Corte IDH sí consideraron la urgencia y riesgo para su vida, y tutelaron su derecho a un tratamiento médico adecuado. Es importante reconocer que dentro del razonamiento de la Corte IDH sobre el daño irreparable, y para resaltar el deber del personal médico de proveer todo el tratamiento que indique la ciencia para proteger a Beatriz, se hace referencia a la consideración de la Sala Constitucional, en la que estima que después de la semana 20 de embarazo "una eventual interrupción del embarazo no conllevaría, ni mucho menos tendría, por objeto, la destrucción del feto y, además que este sería atendido con las medidas necesarias para garantizar, hasta donde fuera posible, su vida extrauterina"15. La anterior consideración sugiere que el acercamiento de la Corte IDH corresponde a la primera lectura sugerida, es decir, a un análisis de ponderación que toma en consideración el desarrollo del no nacido.

\subsection{Protección de la salud mental}

Otro de los aspectos a destacar de las medidas dictadas por la Corte IDH en este asunto, es el reconocimiento de la tutela de la integridad psíquica como determinante en la protección de los derechos a la salud e integridad personal. En efecto, la Corte IDH señala que "además del daño físico que podría producirse en la señora B. el Tribunal resalta que también se estaría poniendo en peligro la salud mental"16.

La Corte IDH en sus consideraciones sobre el posible daño irreparable, analiza los riesgos físicos y emocionales para Beatriz por la continuación del embarazo en relación con su estado de salud. Esta se refiere a los dos riesgos sin distinción. En efecto, al determinar el riesgo en la salud mental, establece que el daño se encuentra ligado a la imposibilidad de actuar de acuerdo a su voluntad, es decir, al no poder ejercer su autonomía reproductiva. La valoración médica encuentra que "en lo que se refiere al estado emocional de la examinada, ésta se encuentra -según lo refiere ella misma- sometida a la presión, ya que se le ha dicho que su vida se encuentra en

\footnotetext{
15 Corte IDH. Asunto B., op.cit., párr. 15.

16 Ibídem, párr.14.
} 
riesgo de muerte si no se decide a 'sacarle al niño'"17. Además, la situación emocional empeora ante la posibilidad de prisión dada la criminalización de la terminación de los embarazos bajo cualquier circunstancia en El Salvador.

Si bien no es explícito en la motivación de la Corte IDH, la protección del derecho a la integridad física y mental, así como del derecho a la salud de Beatriz, se encuentran relacionadas con el derecho a la autonomía reproductiva, desarrollado por la Corte IDH en el caso Artavia Murillo como parte del derecho a la vida privada ${ }^{18}$. Esto, porque la Corte IDH reconoce el daño en la salud mental que produce la imposibilidad de llevar a cabo la decisión de terminar el embarazo para salvar su vida.

\subsection{Derechos reproductivos en el SIDH}

La tutela de los derechos a la vida y a la salud e integridad personal de Beatriz en la situación descrita, solidifican el reconocimiento de los derechos reproductivos en el SIDH.

Los derechos reproductivos solo habían sido abordados de manera tangencial por la Corte IDH hasta la decisión del caso Artavia Murillo. En efecto, antes de dicha decisión, la Corte IDH solo había realizado algunos pronunciamientos, como en el caso Gelman ${ }^{19}$ (sobre la maternidad como parte esencial del libre desarrollo de la personalidad), y había determinado que las muertes maternas eran una vulneración al derecho a la vida en el caso Xákmok Kásek ${ }^{20}$. En este sentido, los precedentes, en su mayoría, provenían de la CIDH en acuerdos de solución amistosa, como los casos de Paulina Ramírez ${ }^{21}$ y María Mamerita Meztanza ${ }^{22}$, así como en pronunciamientos de medidas cautelares (asunto de Amelia en Nicaragua ${ }^{23}$ ) y en informes temáticos, como

17

Ídem.

Corte IDH. Caso Artavia Murillo vs. Costa Rica, op.cit., párrs.146-147. La Corte, al referirse sobre aspectos de la vida privada que se relacionan con el derecho a fundar una familia, el derecho a la integridad física y mental, y específicamente los derechos reproductivos señaló "[E]n segundo lugar, el derecho a la vida privada se relaciona con: i) la autonomía reproductiva, y ii) el acceso a servicios de salud reproductiva, lo cual involucra el derecho de acceder a la tecnología médica necesaria para ejercer ese derecho. El derecho a la autonomía reproductiva está reconocido también en el artículo 16 (e) de la Convención para la Eliminación de todas las Formas de Discriminación contra la Mujer, según el cual las mujeres gozan del derecho ‘a decidir libre y responsablemente el número de sus hijos y el intervalo entre los nacimientos y a tener acceso a la información, la educación y los medios que les permitan ejercer estos derechos'. Este derecho es vulnerado cuando se obstaculizan los medios a través de los cuales una mujer puede ejercer el derecho a controlar su fecundidad. Así, la protección a la vida privada incluye el respeto de las decisiones de convertirse tanto en padre o madre, incluyendo la decisión de pareja de convertirse en padres genéticos. En tercer lugar, la Corte resalta que, en el marco del derecho a la integridad personal, ha analizado algunas situaciones de particular angustia y ansiedad que afectan a las personas, así como algunos impactos graves por falta de atención médica o los problemas de accesibilidad a ciertos procedimientos en salud".

Corte IDH. Caso Gelman vs. Uruguay. Fondo y Reparaciones. Sentencia de 24 de febrero de 2011. Serie C No. 221, párr. 97.

Corte IDH. Caso Xákmok Kásek vs. Paraguay. Fondo y Reparaciones. Sentencia de 24 de agosto de 2010 Serie C No. 214.

CIDH. Paulina del Carmen Ramírez Jacinto vs. México. Informe No.21/07, 2007.

CIDH. Maria Mamerita Meztanza Chavez vs. Perú. Informe No.71/03, 2003.

El 26 de febrero de 2010, la CIDH otorgó medidas cautelares a favor de una persona que la CIDH identificara como Amelia, en Nicaragua. En la solicitud de medida cautelar se alega que Amelia, madre de una niña de 10 años, no estaría recibiendo la atención médica necesaria para tratar el cáncer que padece, en razón de su embarazo. La solicitud añade que los médicos habían recomendado de manera urgente iniciar un tratamiento de quimioterapia o radioterapia, pero que el hospital informó a la madre y los representantes de Amelia que el tratamiento no sería realizado dada la alta posibilidad de que éste provocara un aborto. La Comisión Interamericana solicitó al Estado de Nicaragua que adopte las medidas necesarias para asegurar que la beneficiaria tenga acceso al tratamiento médico que necesite para tratar su cáncer metastático; que adopte estas medidas en concertación con la beneficiaria y sus representantes; y que reserve la identidad de la beneficiaria y de su familia. Dentro del plazo de cinco días 
el "Informe sobre Acceso a Información en Materia de Salud Reproductiva"24, y el "Informe sobre Servicios de Salud Materna desde una Perspectiva de Derechos Humanos"25, entre otros.

El asunto B. solidifica el reconocimiento de los derechos reproductivos en el SIDH ya que, por una parte, consolida los estándares de la CIDH fijados en las medidas cautelares otorgadas en favor de Amelia en Nicaragua y, por otra, porque la protección de los derechos a la vida e integridad personal y a la salud mental de Beatriz se ponen por encima de cualquier otra consideración, lo que implica un reconocimiento al derecho a la autonomía reproductiva. En el asunto de Amelia en Nicaragua, la CIDH ordenó la protección del derecho a la vida e integridad personal de Amelia, a quien se le negaba un tratamiento de quimioterapia, dado que se encontraba embarazada y el tratamiento podía poner en peligro el embarazo. En este sentido, en este caso la Corte IDH reitera estos criterios, privilegiando la protección de la vida y salud de la mujer. Lo relevante es que en este caso, el estándar se fija desde la Corte IDH, lo que implica la obligatoriedad del pronunciamiento -al igual que sus fallos- para el Estado parte y se establece un criterio interpretativo obligatorio para los Estados.

De otra parte, como señalábamos, la protección de los derechos a la vida e integridad personal y a la salud mental de Beatriz, por encima de cualquier otra consideración, implican el reconocimiento al derecho a la autonomía reproductiva. Esto, pues la Corte IDH hace referencia directa a los efectos mentales de no poder acceder a un tratamiento de salud necesario que recae sobre la decisión de terminar un embarazo. Además, el alcance de la protección ordenada por la Corte IDH implica el reconocimiento de que las mujeres no pueden ser estereotipadas por su función reproductiva. En efecto, la protección absoluta al derecho a la vida del que está por nacer por encima de los derechos de la mujer, conlleva el razonamiento de que la mujer tiene -de manera esencial- la función de ser madre, lo que responde a un estereotipo de género ${ }^{26}$. La protección del Estado del derecho a la vida desde esa perspectiva -mediante la penalización total del aborto- pone a la mujer en una situación de vulnerabilidad y discriminación en razón de su género. El razonamiento acerca de la discriminación en razón al género, en conexión con la función reproductiva y como resultado de estereotipos de género, fue establecido por la Corte IDH en el caso Artavia Murillo, y es reiterado de manera implícita en este caso.

\section{Las medidas urgentes y la protección de los derechos reproductivos}

El asunto de Beatriz es un ejemplo de cómo en contextos de criminalización total del aborto, las medidas urgentes pueden ser la única alternativa para proteger la vida y salud de mujeres que enfrentan embarazos de alto riesgo. En ese sentido, en atención a su efectividad, deberían seguir siendo utilizadas en casos similares. Por otra parte, su uso también da visibilidad a las violaciones de derechos humanos que se producen en razón de leyes que no protegen los de-

otorgado para responder, el Estado de Nicaragua informó a la CIDH que se ha iniciado el tratamiento requerido. $\mathrm{CIDH}$. Asunto de "Amelia", medidas cautelares dictadas en contra de Nicaragua, MC 43-10, 2010. Disponible [en línea] <http://www.oas.org/es/cidh/decisiones/cautelares.asp> [consulta: 3 de abril de 2014].

$24 \mathrm{CIDH}$. Acceso a la Información en materia reproductiva desde una perspectiva de derechos humanos. OEA Ser.L/V/II, doc. 61, 2011. Disponible [en línea] <http://www.oas.org/es/cidh/mujeres/docs/pdf/ACCESO\%20INFORMACION\%20MUJERES.pdf> [consulta: 3 de abril de 2014].

$25 \mathrm{CIDH}$. Informe sobre Servicios de salud materna desde una perspectiva de derechos humanos. OEA/Ser.L/V/II, doc.69, 2010. Disponible [en línea] <http://www.oas.org/es/cidh/mujeres/docs/pdf/SaludMaterna2010.pdf> [consulta: 3 de abril de 2014].

26 Un estereotipo es una visión generalizada o una preconcepción sobre los atributos y características de los miembros de un grupo en particular o sobre los roles que tales miembros deben cumplir. Los estereotipos de género se refieren a la construcción social y cultural de hombres y mujeres, en razón de sus diferentes funciones físicas, biológicas, sexuales. Véase: COOK, Rebecca y CUSACK, Simone. Estereotipos de género. Perspectivas Legales Transnacionales. Colombia: Profamilia, University of Pennsylvania Press, 2010. 
rechos de las mujeres, lo que ayuda desarrollar el entendimiento de las obligaciones estatales que se desprenden de la CADH desde una perspectiva de los derechos de la mujer. Asimismo, la utilización de las medidas cautelares y provisionales en estos casos, abre paso a la creación de precedentes sobre derechos reproductivos que tengan un impacto directo en la protección del derecho a la igualdad de las mujeres; en efecto, el desarrollo de estos precedentes coadyuva a que los Estados estén obligados a adecuar su derecho interno en conformidad a los estándares desarrollados por la jurisprudencia interamericana. 
